\title{
Link-Sign Prediction in Signed Directed Networks from No Link Perspective
}

\author{
Quang-Vinh Dang ${ }^{0000-0002-3877-8024}$ \\ Data Innovation Lab, Industrial University of Ho Chi Minh city, Ho Chi Minh, \\ Vietnam
}

\begin{abstract}
Predicting future sign of connections in a network is an important task for online systems such as social networks, e-commerce and other services. Several research studies have been presented since the early of this century to predict either the existence of a link in the future or the property of the link. In this study we present a new approach that combine both families by using machine learning techniques. Instead of focusing on the established links, we follow a new research approach that focusing on no-link relationship. We aim to understand the move between two states of no-link and link. We evaluate our methods in popular real-world signed networks datasets. We believe that the new approach by understanding the no-link relation has a lot of potential improvement in the future.
\end{abstract}

Keywords: Signed Network · Machine learning · Link Prediction.

\section{Introduction}

Many real-world systems can be modelled by a signed-directed graph [17], such as connection graph between users on social networks like Facebook, citation graphs in scientific publication, recommendation graph in e-commerce, or the activities like view/like/dislike graphs on YouTube.

In order to improve the quality of user experience in these systems and increase the traffic usage hence the revenue of the systems, the service providers usually provide a recommendation service to suggest users what service to use next. Definitely the recommendation engine should be able to understand the users to recommend the service that the user want to use or the item that the user want to buy. Besides the well-known collaborative filtering technique, another approach to look at the problem is to consider the recommendation as the link-attribute prediction problem [23], i.e. we would like to predict the property of the link between two nodes. For instance, in e-commerce systems like Amazon, if we consider buyers and items are nodes and connections between them are the rating scores the buyers give to the items, link-attribute prediction shares a same goal with collaborative filtering.

In this study, we limit to the problem of link-sign prediction, i.e. we consider only two possible attributes of a link which are positive or negative [36]. We can think of positive links as a node (user on Facebook, buyer on 
Amazon, viewer on YouTube) like another node, and negative links as dislike relation. If we can predict accurately the signs of the links, we can improve significantly the quality of the recommender systems [16].

The problem of link-sign prediction has attracted a lot of studies in the last two decades. However, the problem is divided into link-prediction and sign prediction separately. In this study we consider unifying the two problems into one single framework.

\section{Related Works}

The problem of link-sign prediction is usually divided into the link-prediction problem [41] and the sign-prediction problem [35]. The link-predictor aims to predict the existence of the link in the future, given the non-existence of this link at present. The sign-predictor aims to predict the sign of a link in the future, given that the existence of the link is confirmed. The difference is visualized in Figure 1.

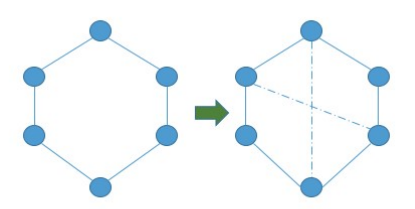

(a)

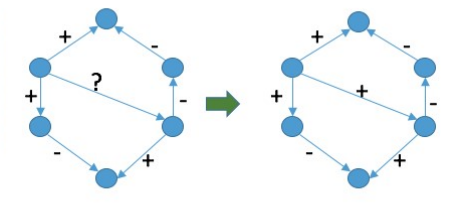

(b)

Fig.1. (a) Link-prediction; and (b) Sign-prediction

\subsection{Link-Prediction Problem}

Link-prediction can be roughly divided into proximity-based and learningbased methods [40].

Proximity-based methods mostly rely on homophily theory [45] which states that similar users tend to connect to each other's. The proximity metrics can be nodal proximity-based metrics [61] or structural proximity-based metrics [41]. The nodal-based proximity methods use the personal profile information of the nodes to design the proximity metric. For instance, the authors of [60] proposed to use demographics information such as age, education and occupation. In [58] the authors also used geographic information in physical space to estimate the 
distances between two nodes in cyber-space. Several studies employed user activities on social networks such as keywords, hashtags or semantic of the content that the users share or view on social network to calculate their similarity [52, 44,64]. Among different metrics designed in different studies, cosine similarity is mostly used as the proximity metric $[58,9,40]$. Other popular distance metrics have been used in literature are KL-divergence [52] or Jaccard's coefficient [51, 50].

Structural-based methods do not rely on the individual information of the nodes but based on the topological information of the graph [41,21]. An early attempt is credited to [1] that defines the Adamic-Adar measure between node $i$ and node $j$ as:

$$
A A_{i j}=\sum_{v_{z} \in \Gamma_{i} \cap \Gamma_{j}} \frac{1}{\log \left|\Gamma_{i} \cap \Gamma_{j}\right|}
$$

where $\Gamma_{i}$ is the set of direct neighbors of the node $i$. The metric is inspired by the Erdos-Renyi model [5]. Both theoretical and empirical studies showed that the likelihood of a link depends on the the size of direct neighborhood of a node [46,41]. Other well-known structural-based measures that rely on neighborhood information are SimRank [32], Sorensen Index [54] or HP/HD Index [49].

Another kind of structural-based proximity is to rely not on the neighborhood information but on the connection between two nodes. The core idea is to calculate the distance between two nodes as the surrogate estimation of how close they are to each other, hence how likely that they will connect. One of very early approaches belongs to [33] as:

$$
\underset{k}{K Z_{i j}}=\underset{\mathrm{X}}{\mathrm{X}} k \mid \text { path }<i j k>1
$$

The core idea of Katz index [33] is to combine all connected paths between two nodes with associated weights as their corresponding length. The authors of [67] updated the Katz index to use local-path information. Other studies [26, 57] modified the PageRank algorithm for the link-prediction problem. Recently, [62] added the interaction among paths, i.e. the authors consider the interaction between nodes that belong to different paths into consideration, to achieve a better prediction. [10] explored a very new and interesting aspect of 
the network which is the uncertainty of the network topology, i.e. when we are not $100 \%$ sure if there is a link or no-link between two nodes.

On the other hand, learning-based link-prediction methods build machine learning models to predict the probability that a link will be established in the future. In order to build the machine learning models, features needed to be extracted. The features might be similar to the similarity metrics described above, i.e. neighbor-based features such as common friends [6] or path-based features such as the shortest path between two nodes [42]. The most common algorithms are logistic regression [47] or SVM [3]. Most recently the authors of [65] proposed to use SVM classification method on Bhattacharyya kernel [31] for the task of link-prediction.

\subsection{Sign-Prediction Problem}

In contrast to link-prediction problem where the problem is formed as predicting whether a link will be established in the future or not, the signprediction problem tries to predict the attribute of the link, here positive or negative sign, given the existence of the link in the future [23].

Early attempts in sign-prediction used rule-based systems, such as "friends of friends are friends" and "friends of enemies are enemies" for prediction [23]. In order to speed up the calculation, the prediction is performed as matrix multiplication. The following studies turned the problem into a low-rank matrix factorization problem [43] as in the recommender systems.

Starting with the work of [36,35], structural balance theory and social status theory are used to interpret the structure of the social graphs hence to predict the sign of the connections. We visualized two theories in Figure 2 and 3 [17, 13]. Several studies have followed the work of [35] such as [11] by extending the cycle length or [27] by integrating the social theories into matrix factorization techniques. In the same direction, [34] focused on micro-structure of three users with bidirectional links and their similarities, for the sign prediction.

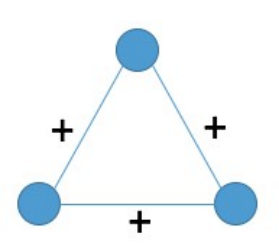

(a)

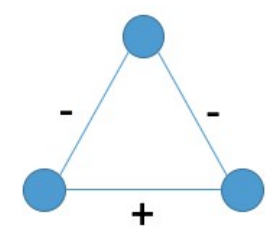

(b)

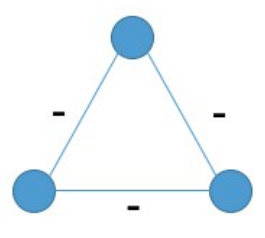

(c)

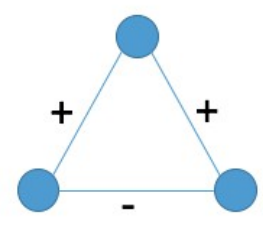

(d) 
Fig.2. Visualization of structural balance theory [35]. Structural balance theory considers the triads (a) and (b) are balanced, while (c) and (d) are not. The weak balance theory considers (c) as balanced as well, regardless the direction.

The authors [53] argued that, (i) even structural balance theory and social status theory played an important role in existing research studies on link sign prediction, they are not scalable up to the size of the modern networks, and (ii) a fully observed network is usually not available in practice. They develop Bayesian node features based on partially observed networks and used a logistic regression classifier for link sign prediction. However, the obtained performance is weak compared to other recent studies.

Graph embedding techniques attracted a lot of attention recently [22,59, 28]. Many graph embedding research studies are inspired by word embedding techniques [48] as they share a similar goal. The authors of [17] combined word embedding technique, random walk and Long-Short Term Memory (LSTM) networks to utilize the time information of the network for sign-prediction.

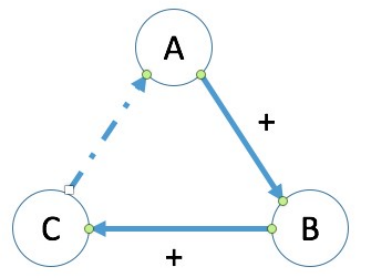

Fig.3. The sign of the dash line from $C$ to $A$ is inferred by their social status. Because

$A \rightarrow B$ and $B \rightarrow C$, therefore hence the social status theory states that $B>A$ and $C>B$, so $C>$ $A$, leads to the prediction that the sign of line from $C$ to $A$ is negative.

As pretrained models are more and more popular in natural language processing community and achieve a lot of success, transfer learning has been used in sign-prediction as well. This direction has a lot of potential usage as it does not require labelled data [66].

Most existing works in sign-prediction assumes the previous task which is link-prediction has been done, so they assume the existence of the link and focus only on the sign of the link. 


\section{Understanding No-Link}

As we discussed in Section 2, the work of link-sign prediction is usually divided into two steps: link-prediction and sign-prediction [38]. Given that the performance of state-of-the-art link-prediction algorithms are far from perfect [40], it is not realistic to expect a high-accuracy prediction in sign-prediction if we consider the task link-sign prediction as an entire task.

Given that the majority of a network should be no-link [17], it is important to understand the no-link relationship to achieve a higher-accuracy prediction. Hence we focus on the move from no-link into link between two nodes. We collected features based on sociology theories and other studies [38] such as Balance Theory [4], Status Theory [18], Reciprocity [20] or Frequent Subgraph [35]. We also derived features automatically through some graph embedding techniques such as DeepWalk [48], LINE [55] and node2vec [22]. We try to capture the semantic of the network just before a link is established. Our approach shares some similarities with the work of [39] as we combine both explicit and implicit features, but different from [39] we treated different links with different weights. Furthermore, in [39] the authors simply add two explicit and latent scores then solving an optimization problem, so they consider two sets of features as the same weight without any learning process.

Regarding the no-link relations, we consider the time when a node decides to make a link to a particular other node but not other nodes. At this very point of time we consider that, it means the first node decides to not make a link to other nodes. We collected the same features but with label of no-link.

Instead of treating the problem as the traditional classification problem [35, $23,63,17]$ we convert the problem into the ranking problem. We use a stateofthe-art ranking algorithm implemented in XGBRanker version 1.0.0 ${ }^{1}$ which has just been released at the time of writing. In our opinion, this approach makes more sense as what we need to optimize in practice, such as in an e-commerce system, is the ranking metric, i.e. which item a user will most likely like, rather than a normal classification problem [38]. Indeed we can use the output of any classification problem as the ranking score but actually they are optimized for different purposes [37].

1 https://xgboost.readthedocs.io 


\section{Experiments}

\subsection{Datasets}

We used well-known signed network datasets that have been used widely in the literature: Epinions, Slashdot and Wikipedia RfA [2,17,35,63]. They are realworld signed networks collected from e-commerce website (Epinions), sharing news website (Slashdot) or crowd-sourcing encyclopedia (Wikipedia). In these websites, users can form explicitly trust (positive) / distrust (negative) links to other users. The connections are provided with the established time that allow us to understand the history of the network.

We display some basic statistics of the dataset in Table 1 [17].

\begin{tabular}{cccc}
\hline & Epinions & \multicolumn{2}{c}{ Slashdot Wikipedia } \\
\hline \# of nodes & 119217 & 82140 & 7118 \\
\# of edges & 841200 & 549202 & 103747 \\
fraction of edges & $6 e-5$ & $8 e-5$ & $2 e-3$ \\
+ edges (\%) & 85.0 & 77.4 & 78.8 \\
- edges (\%) & 15.0 & 22.6 & 21.2 \\
largest WCC (\%) & 99.1 & 100 & 100 \\
average \# of directed connection & 590 & 327 & 418 \\
\# of triads & 133754071508105 & 790532 \\
fraction of triads & $1.35 e-105.46 e-11$ & $4.25 e^{-9}$
\end{tabular}

Table 1. Basic statistics of datasets. WCC stands for weakly connected component [19].

The authors of [39] studied the change from no-link to link between two nodes given the they have common neighbors. The analysis is presented in Figure 4 for Epinions dataset. It is agreed between several recent studies [39,38] that the process of moving from no-link to link is really important.

For each dataset, we divide the train/validation/test set according to the ratio of $60: 20: 20$ by temporal order, i.e. we use $60 \%$ of the links that are 


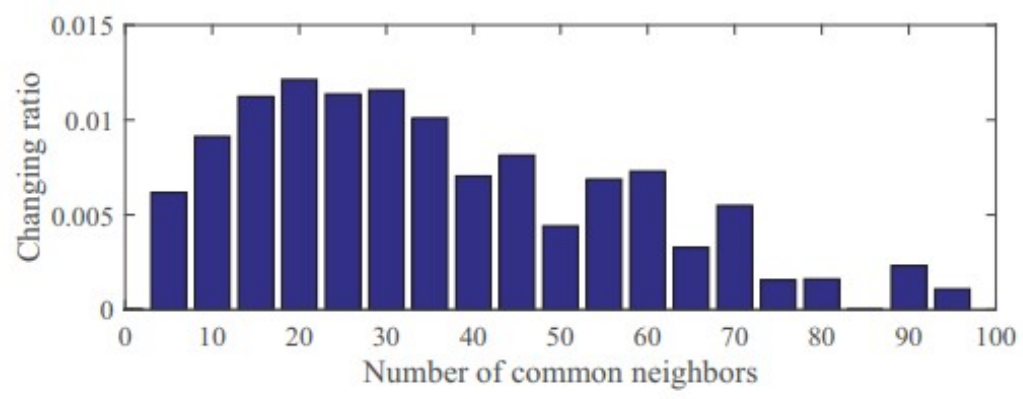

Fig.4. Changing ratio given the common neighbors between two nodes [39]. The X-axis represents the number of common neighbors in 15 months and the $\mathrm{Y}$-axis represents the proportion of two nodes that actually formed a link.

established first in each network as the training set, $20 \%$ of the links that are established next for the validation set, and $20 \%$ of the links that are established last for testing purpose.

In order to avoid overfitting, we apply early stopping technique [8]. Other hyper-parameters are optimized by using Bayesian optimization [7].

\subsection{Evaluation Metric}

As the matter of fact, the network in general is extremely balanced: most of the potential links do not exist. As we present in Table 1, there is around only less than $0.1 \%$ of the potential links in the network, i.e. a connection that might be exist between any two nodes, is actually established in a network. It is understandable because usually a typical person can only make a few connections, and even if the degree of a node is thousands it is still a very small number compared to billions of nodes in modern networks like Facebook.

Definitely some popular metrics which are sensitive to the imbalanced dataset such as accuracy score or even F-score are not usable to evaluate link prediction problem [30]. AUC is used more widely [17] but the AUC is designed only for a binary classification problem [29]. In this paper we will use a more generalized AUC metric designed by [25] which has been adapted in multi-class classification problem [14,15].

The generalized $A U C$ [25] is defined as follows. Assume that there is a multiclass classification problem with $c>2$ classes, notated as $0,1, \ldots, c-1$. We define $A^{\wedge}(i \mid j)$ as the probability that a randomly drawn member of class $j$ will have a lower estimated probability of belonging to class $i$ than a random 
member of class $i$. We define $A^{\wedge}(i, j)=\left[A^{\wedge}(i \mid j)+A^{\wedge}(j \mid i)\right] / 2$. We calculate the value of $A U C$ as:

$$
A U C=\frac{2}{c(c-1)} \sum_{i<j} \hat{A}(i, j
$$

The generalized AUC is chosen because it is based on the same principle of measuring the ranking order as in typical AUC [12]. Similar to typical AUC, the generalized AUC ranges from 0.0 to 1.0, higher is better.

\subsection{Experimental Results}

We compare our method with several other baseline methods, such as multiclass classification [17]. We note that it is our method is not comparable to existing works because it focuses on both link-prediction and sign-prediction at the same time.

Here, we consider the problem of predicting at a given point of time, given that there is no link between node $A$ and node $B$, which action will be performed by node $A$ : (i) establish a positive link, or (ii) establish a negative link, or (iii) do not establish a link.

\begin{tabular}{cccc}
\hline Methods & \multicolumn{3}{c}{ Epinions Slashdot Wikipedia } \\
\hline Classification & 0.72 & 0.74 & 0.77 \\
Ranking & 0.74 & 0.75 & 0.79 \\
\hline
\end{tabular}

Table 2. Generalized AUC of our method in different datasets.

The result is presented in Table 2. We notice that in general the ranking approach achieve a better performance, which is confirmed by other research studies [24] as the AUC by its nature is optimized by a ranking method.

Similar to other link-prediction [35] or sign-prediction work [17], both methods perform best in Wikipedia dataset. The reason might be the topological structure of Wikipedia that is much more dense than other networks, but we let it be an open question for further research works.

\section{Conclusions}

In this study we presented our work on link-sign prediction in signed networks. Together with the preliminary work of [38], they are very first working in considering link-prediction and sign-prediction into one problem. By combining the problems, we eliminate the accumulated error over multiple 
prediction steps. We note that the approach presented in this paper is a topological-based approach [56], i.e. we only rely on the features that can be derived from the topology of the network and we do not use any personal information of node such as gender or activity history.

In the future research we plan to incorporate more temporal features as described in [17]. We believe that by understanding the movement from no-link to a link in a network we will gain more insights of the link-sign prediction problem. In other words, we would like to know what bring a node to a very precise point of time before he or she decide to form a link or not, not only just before a connection is made. Another interesting research direction is to predict the time when a link is made rather than considering this time as a given input.

\section{References}

1. Adamic, L.A., Adar, E.: Friends and neighbors on the web. Social Networks 25(3), 211-230 (2003)

2. Agrawal, P., Garg, V.K., Narayanam, R.: Link label prediction in signed social networks. In: IJCAI. pp. 2591-2597. IJCAI/AAAI (2013)

3. Al Hasan, M., Chaoji, V., Salem, S., Zaki, M.: Link prediction using supervised learning. In: SDM (2006)

4. Antal, T., Krapivsky, P.L., Redner, S.: Social balance on networks: The dynamics of friendship and enmity. Physica D: Nonlinear Phenomena 224(1-2), 130-136 (2006) 5. Baraba'si, A.L., et al.: Network science. Cambridge university press (2016)

6. Benchettara, N., Kanawati, R., Rouveirol, C.: A supervised machine learning linkprediction approach for academic collaboration recommendation. In: RecSys. pp. 253-256. ACM (2010)

7. Bergstra, J., Komer, B., Eliasmith, C., Yamins, D., Cox, D.D.: Hyperopt: a python library for model selection and hyperparameter optimization. Computational Science \& Discovery 8(1), 014008 (2015)

8. Caruana, R., Lawrence, S., Giles, C.L.: Overfitting in neural nets: Backpropagation, conjugate gradient, and early stopping. In: NIPS. pp. 402-408. MIT Press (2000)

9. Chen, J., Geyer, W., Dugan, C., Muller, M.J., Guy, I.: Make new friends, but keep the old: recommending people on social networking sites. In: CHI. pp. 201-210. ACM (2009)

10. Chen, X., Guo, J., Pan, X., Zhang, C.: Link prediction in signed networks based on connection degree. J. Ambient Intelligence and Humanized Computing 10(5), 1747-1757 (2019)

11. Chiang, K., Natarajan, N., Tewari, A., Dhillon, I.S.: Exploiting longer cycles for link prediction in signed networks. In: CIKM. pp. 1157-1162. ACM (2011)

12. Cortes, C., Mohri, M.: AUC optimization vs. error rate minimization. In: NIPS.pp. 313-320. MIT Press (2003)

13. Dang, Q.V.: Trust assessment in large-scale collaborative systems. (Evaluation' de la confiance dans la collaboration a' large 'echelle). Ph.D. thesis, University of Lorraine, Nancy, France (2018)

14. Dang, Q.V., Ignat, C.L.: Measuring quality of collaboratively edited documents: The case of Wikipedia. In: CIC. pp. 266-275. IEEE Computer Society (2016) 
15. Dang, Q.V., Ignat, C.L.: Quality assessment of Wikipedia articles without feature engineering. In: JCDL. pp. 27-30. ACM (2016)

16. Dang, Q.V., Ignat, C.L.: dTrust: A simple deep learning approach for social recommendation. In: CIC. pp. 209-218. IEEE (2017)

17. Dang, Q., Ignat, C.: Link-sign prediction in dynamic signed directed networks. In: CIC. pp. 36-45. IEEE Computer Society (2018)

18. Davis, J.A., Leinhardt, S.: The structure of positive interpersonal relations in smallgroups. (1967)

19. Easley, D., Kleinberg, J.: Networks, crowds, and markets. CUP (2010)

20. Falk, A., Fischbacher, U.: A theory of reciprocity. Games and Economic Behavior 54(2), 293-315 (2006)

21. Fan, C., Li, D., Teng, Y., Fan, D., Ding, G.: Exploiting non-visible relationship inlink prediction based on asymmetric local random walk. In: ICONIP (5). Lecture Notes in Computer Science, vol. 10638, pp. 731-740. Springer (2017)

22. Grover, A., Leskovec, J.: node2vec: Scalable feature learning for networks. In: KDD (2016)

23. Guha, R.V., Kumar, R., Raghavan, P., Tomkins, A.: Propagation of trust and distrust. In: WWW. pp. 403-412. ACM (2004)

24. Güvenir, H.A., Kurtcephe, M.: Ranking instances by maximizing the area under ROC curve. IEEE Trans. Knowl. Data Eng. 25(10), 2356-2366 (2013)

25. Hand, D.J., Till, R.J.: A simple generalization of the area under the ROC curve for multiple class classification problems. Machine Learning 45(2), 171-186 (2001)

26. Haveliwala, T.H.: Topic-sensitive pagerank. In: WWW. pp. 517-526. ACM (2002) 27. Hsieh, C., Chiang, K., Dhillon, I.S.: Low rank modeling of signed networks. In: KDD. pp. 507-515. ACM (2012)

28. Hu, B., Wang, H., Yu, X., Yuan, W., He, T.: Sparse network embedding for community detection and sign prediction in signed social networks. J. Ambient Intelligence and Humanized Computing 10(1), 175-186 (2019)

29. Huang, J., Ling, C.X.: Using AUC and accuracy in evaluating learning algorithms. IEEE Trans. Knowl. Data Eng. 17(3), 299-310 (2005)

30. Japkowicz, N., Shah, M. (eds.): Evaluating Learning Algorithms: A ClassificationPerspective. Cambridge University Press (2011)

31. Jebara, T., Kondor, R.: Bhattacharyya expected likelihood kernels. In: COLT. Lecture Notes in Computer Science, vol. 2777, pp. 57-71. Springer (2003)

32. Jeh, G., Widom, J.: Simrank: a measure of structural-context similarity. In: KDD.pp. 538543. ACM (2002)

33. Katz, L.: A new status index derived from sociometric analysis. Psychometrika18(1), 39-43 (1953)

34. Khodadadi, A., Jalili, M.: Sign prediction in social networks based on tendency rate of equivalent micro-structures. Neurocomputing p. 10 (2017)

35. Leskovec, J., Huttenlocher, D.P., Kleinberg, J.M.: Predicting positive and negative links in online social networks. In: WWW. pp. 641-650. ACM (2010)

36. Leskovec, J., Huttenlocher, D.P., Kleinberg, J.M.: Signed networks in social media. In: CHI. pp. 1361-1370. ACM (2010)

37. Li, H.: Learning to Rank for Information Retrieval and Natural Language Processing, Second Edition. Synthesis Lectures on Human Language Technologies, Morgan \& Claypool Publishers (2014)

38. Li, X.: Towards practical link prediction approaches in signed social networks. In: UMAP. pp. 269-272. ACM (2018) 
39. Li, X., Fang, H., Zhang, J.: FILE: A novel framework for predicting social status in signed networks. In: AAAI. pp. 330-337. AAAI Press (2018)

40. Li, Z.L., Fang, X., Sheng, O.R.L.: A survey of link recommendation for social networks: Methods, theoretical foundations, and future research directions. ACM Trans. Management Inf. Syst. 9(1), 1:1-1:26 (2018)

41. Liben-Nowell, D., Kleinberg, J.M.: The link-prediction problem for social networks. JASIST (2007)

42. Lichtenwalter, R.N., Lussier, J.T., Chawla, N.V.: New perspectives and methodsin link prediction. In: KDD. pp. 243-252. ACM (2010)

43. Ma, H., Yang, H., Lyu, M.R., King, I.: Sorec: social recommendation using probabilistic matrix factorization. In: CIKM. pp. 931-940. ACM (2008)

44. Makrehchi, M.: Social link recommendation by learning hidden topics. In: RecSys.pp. 189196. ACM (2011)

45. McPherson, M., Smith-Lovin, L., Cook, J.M.: Birds of a feather: Homophily in social networks. Annual review of sociology 27(1), 415-444 (2001)

46. Newman, M.E.: Clustering and preferential attachment in growing networks. Physical review E 64(2), 025102 (2001)

47. O’Madadhain, J., Hutchins, J., Smyth, P.: Prediction and ranking algorithms for event-based network data. ACM SIGKDD explorations newsletter 7(2), 23-30 (2005)

48. Perozzi, B., Al-Rfou, R., Skiena, S.: Deepwalk: online learning of social representations. In: KDD. pp. 701-710. ACM (2014)

49. Ravasz, E., Somera, A.L., Mongru, D.A., Oltvai, Z.N., Baraba'si, A.L.: Hierarchicalorganization of modularity in metabolic networks. Science 297(5586), 15511555 (2002)

50. Scellato, S., Noulas, A., Mascolo, C.: Exploiting place features in link prediction on location-based social networks. In: KDD. pp. 1046-1054. ACM (2011)

51. Schifanella, R., Barrat, A., Cattuto, C., Markines, B., Menczer, F.: Folks in folksonomies: social link prediction from shared metadata. In: WSDM. pp. 271-280. ACM (2010)

52. Shen, D., Sun, J., Yang, Q., Chen, Z.: Latent friend mining from blog data. In: ICDM. pp. 552-561. IEEE Computer Society (2006)

53. Song, D., Meyer, D.A.: Link sign prediction and ranking in signed directed social networks. Social Netw. Analys. Mining 5(1), 52:1-52:14 (2015)

54. Sørensen, T.J.: A method of establishing groups of equal amplitude in plant sociology based on similarity of species content and its application to analyses of the vegetation on Danish commons. I commission hos E. Munksgaard (1948)

55. Tang, J., Qu, M., Wang, M., Zhang, M., Yan, J., Mei, Q.: LINE: large-scale information network embedding. In: WWW. pp. 1067-1077. ACM (2015)

56. Tang, J., Chang, Y., Aggarwal, C., Liu, H.: A survey of signed network mining in social media. ACM Comput. Surv. 49(3), 42:1-42:37 (2016)

57. Tong, H., Faloutsos, C., Pan, J.Y.: Fast random walk with restart and its applications. In: ICDM. pp. 613-622. IEEE (2006)

58. Wang, D., Pedreschi, D., Song, C., Giannotti, F., Baraba'si, A.: Human mobility, social ties, and link prediction. In: KDD. pp. 1100-1108. ACM (2011)

59. Wang, H., Zhang, F., Hou, M., Xie, X., Guo, M., Liu, Q.: SHINE: signed heterogeneous information network embedding for sentiment link prediction. In: WSDM. pp. 592-600. ACM (2018)

60. Xu, Y., Rockmore, D.N.: Feature selection for link prediction. In: PIKM. pp. 25-32. ACM (2012) 
61. Yang, Y., Chawla, N.V., Sun, Y., Han, J.: Predicting links in multi-relational andheterogeneous networks. In: ICDM. pp. 755-764. IEEE Computer Society (2012)

62. Yao, Y., Zhang, R., Yang, F., Tang, J., Yuan, Y., Hu, R.: Link prediction in complexnetworks based on the interactions among paths. Physica A: Statistical Mechanics and its Applications 510, 52-67 (2018)

63. You, Q., Wu, O., Luo, G., Hu, W.: A probabilistic matrix factorization method for link sign prediction in social networks. In: MLDM. Lecture Notes in Computer Science, vol. 9729, pp. 415-420. Springer (2016)

64. Yuan, G., Murukannaiah, P.K., Zhang, Z., Singh, M.P.: Exploiting sentiment homophily for link prediction. In: RecSys. pp. 17-24. ACM (2014)

65. Yuan, W., He, K., Guan, D., Zhou, L., Li, C.: Graph kernel-based link prediction for signed social networks. Information Fusion 46, 1-10 (2019)

66. Yuan, W., Pang, J., Guan, D., Tian, Y., Al-Dhelaan, A., Al-Dhelaan, M.: Sign prediction on unlabeled social networks using branch and bound optimized transfer learning. Complexity 2019, 4906903:1-4906903:11 (2019)

67. Zhou, T., Lü, L., Zhang, Y.C.: Predicting missing links via local information. The European Physical Journal B 71(4), 623-630 (2009) 